was sketched in a detailed Report communicated by me to the Geological Society on April 25, 1888. My friend Prof. Lapworth has no scientific comrade who has more frankly and practically acknowledged his great geological achievements than I have done. January 23, 1893 .

\section{The Identity of Energy.}

I AM glad to see that in the introduction to his severelydifficult memoir, published in the Philosophical Transactions for 1892, "On the Forces, Stresses, and Fluxes of Energy in the Electromagnetic Field" (p. 427), Mr. Oliver Heaviside notices and criticizes some ideas of mine, published in the Philosophical Magazine for June 1885 and other places, concerning energy.

The statements I then made, and to which I still rigidly hold, are ( $\mathrm{I}$ ) that energy has identity like matter, and not merely conservation ; (2) that whenever energy is transferred from one body to another, it is also transformed from potential to kinetic, or vice versâ.

The basis of the first assertion is the fact that energy is always passed on continuously through space, i.e. that its transfer occurs along a definite path, instead of merely appearing in one place and disappearing in another.

The law of conservation would be satisfied by disappearance and equal reappearance ; the law of identity requires a continuous act of transfer. The latter is true for matter, and I assert that by thinking of a number of instances, it will be perceived true for energy. In all mechanical instances, as of belts and shafting, the transfer of energy is obvious ; it was not so obvious in electromagnetic actions, between dynamo and motor for instance, until Prof. Poynting clearly demonstrated that it was in accordance with Maxwell's principles.

Mr. Heaviside objects that we are not able to assert it for gravitational energy. Well, that depends on what view we take of gravitation; but I submit that until something more is certainly known about it, the safest plan is not to assert, but to assume, that in this case also what is known in every other case likewise occurs, and to trace the consequences of the hypothesis in the hope that it may lead to some conclusion verifiable or falsifiable by experiment. The reason I attach importance to this doctrine of the identity or continuity of transfer of energy is because it greatly simplifies the fundamental mechanical laws, and emphasizes without risk of vagueness the denial of action at a distance.

If action at a distance (no matter how minute) can ever occur, then indeed the continuous transfer of energy breaks down. But observe that there is no necessity for the transfer to occur at a finite velocity in order to avoid action at a distance, i.e. action without a medium. By the thrust of an incompressible pole, energy is transferred from butt to tip, just as really as if the compressed and recoiling layers could be demonstrated and its velocity measured. So likewise the pull of gravitation may be (and pro tem. I believe is) transmitted by an incompressible (or nearly incompressible) ether, so that the force is felt instantaneously (or nearly instantaneously) at all distances where matter exists ; but that by no means militates against a genuine act of transfer. The conservation of matter makes experiments on gravitation difficult; if we could suddenly create or destroy a piece of matter there might be some remote chance of determining the rate at which its gravitative influence was felt. Especially if by alternately generating and destroying it we could set up a series of waves of perhaps measurable length.

And although this is as yet impossible, many known facts lead us to conclude that if gravitation has any velocity at all short of infinite, it is at least immensely greater than the speed of light. And seeing that the one phenomenon is concerned with the transverse (electric) elasticity of ether, and the other with its longitudinal elasticity, there is nothing surprising in that.

By all means, however, as Mr. Heaviside urges, let gravitation be included in general etherial equations whenever possible; and it may perhaps be wise to assume some unknown finite rate of propagation and trace its consequences with the object of verifying or disproving them.

So far as I understand, however, this is not unlike what Helmholtz did, by his generalization of Maxwell's electromagnetic theory; with the result that the course of experiment so far has been to justify the simple Maxwellian theory, and to make the longitudinal ether thrust velocity practically infinite.
And now for the second assertion, that whenever energy is transferred from one body to another, it is also transformed, and vice versa $\hat{a}$. This is to me not an opinion, but a demonstrated theorem (as has been shown in the paper referred to); but it must be understood in what sense I consistently use the word body in this connection. I do not necessarily mean a visible lump of matter. The molecules of a lump are to be regarded as a different "body" to the whole mass; and again, the ether everywhere embathing them is another distinct "body."

But so long as a piece of matter is merely moving through space with all the energy it may happen to contain, I do not consider that a transfer at all. There is a transfer of energy in one sense, viz, that of locomotion, but there is no transfer from one body to another except when work is done at their point of contact, and energy gained by one and lost by the other, being transferred across their common boundary surface. In al such cases of "activity" the energy transferred is necessarily in the first instance transformed; though by means of another transfer it may very speedily be transformed back again; and so speedily sometimes is the re-transformation effected that the intermediate condition has a tendency to get overlooked. In wave-motion a transfer and transformation occurs during every quarter period.

Mr. Heaviside seems to think that the mere convection of energy should be included as one kind of transfer; but surely that is scarcely convenient? So long as energy retains its form and adherence 10 one body, so long there is no true activity; no work is being done-the energy is simply stored. It may be stored in a bent spring, or in a flying bullet, or in a revolving fly-wheel. It is impossible to have kinetic energy at all without convection, and a distinction must be drawn between the mere existence of energy and the active and useful flux or transfer of the same.

Mr. Heaviside further seems to consider circuital fluxes of energy as strange and useless phenomena. But $I$ see no reason in this at all. The circulation of matter-for instance in the inner circle of the Metropolitan railway-is, I suppose, considered useful. The circulation of commodities is the essence of commerce. So does the circulation of energy constitute the activity of the material universe. It is the act of transfer that is beneficial (or the reverse); what becomes of a conservative quantity is a minor matter. It must go somewhere, and may very well, after a series of transfers, ultimately return to its starting point. [Parenthetically I should like to preach here against what I hold to be the pernicious doctrine of (at least amateur) political economists, that because money locally spent is not destroyed, but remains in the community, it does not much matter how much transferring power is permitted or granted to one individual, - as if the money itself were the useful commodity, and not the power of determining its direction of transfer or non-transfer. The control of every transfer should be jealously watched, for that is the greedily-desired power.]

So long as circuital convection of energy goes on without transfer-as, for instance, in the rim of a non-working flywheel-so long the energy is merely stored; but directiy a belt is fitted on with different tensions in its two halves, a portion of the energy is tangentially tapped off, and transfer and activity begin. The kinetic energy of the wheel is converted into strain or stress energy of the belt, which then by simple locomotion passes it on to something else. I perceive, however, that there is a slight difficulty about this simple case of locomotive conveyance of stress energy by a really inelastic substance; but only because the details of any infinitely rapid process are difficult to follow. I perceive moreover that in many cases it is not worth while to attend to the alternate compressions and motions which constitute a longitudinal pulse, and that the idea of simple locomotion may be conveniently introduced to cover the case of a stressed body moving; but the convenience is I think only attained by shutting our eyes to the essential processes which in all actual matter must be occurring.

I trust that $\mathrm{Mr}$. Heaviside may find time to notice this letter, and attack anything he disagrees with, in order that the whole matter may become thoroughly clear. OLIVER LoDGE.

\section{A Proposed Handbook of the British Marine Fauna.}

I AM obliged to Prof. Thompson for his criticism of my scheme, although only one of the points he raises is new to me as I think it will be to most zoologists-viz. that "there are no nematophores on the stem "in Antennularia. I thought $A$. NO. I 2 I 3 , VOL. 47 ] 